\title{
High resolution chromosome 3p, 8p, 9q and 22q allelotyping analysis in the pathogenesis of gallbladder carcinoma
}

\author{
II Wistuba*, , A Maitra², R Carrasco', M Tang', P Troncoso', JD Minna ${ }^{3}$ and AF Gazdar \\ 'Department of Anatomic Pathology, Pontificia Universidad Catolica de Chile, Marcoleta 367, P.O. Box 1/4-D, Santiago, Chile; ${ }^{2}$ Department of Pathology, \\ Johns Hopkins Medical Institutions, Baltimore, Maryland, MD 21209, USA; ${ }^{3}$ Hamon Center for Therapeutic Oncology Research, UT Southwestern \\ Medical Center, Dallas, Texas, TX 75390, USA
}

Our recent genome-wide allelotyping analysis of gallbladder carcinoma identified 3p, 8p, 9q and 22q as chromosomal regions with frequent loss of heterozygosity. The present study was undertaken to more precisely identify the presence and location of regions of frequent allele loss involving those chromosomes in gallbladder carcinoma. Microdissected tissue from 24 gallbladder carcinoma were analysed for PCR-based loss of heterozygosity using 81 microsatellite markers spanning chromosome 3p $(n=26), 8 p(n=14), 9 q(n=29)$ and 22q $(n=12)$ regions. We also studied the role of those allele losses in gallbladder carcinoma pathogenesis by examining 45 microdissected normal and dysplastic gallbladder epithelia accompanying gallbladder carcinoma, using 17 microsatellite markers. Overall frequencies of loss of heterozygosity at 3p (I00\%), 8p (I00\%), 9q (88\%), and 22q (92\%) sites were very high in gallbladder carcinoma, and we identified 13 distinct regions undergoing frequent loss of heterozygosity in tumours. Allele losses were frequently detected in normal and dysplastic gallbladder epithelia. There was a progressive increase of the overall loss of heterozygosity frequency with increasing severity of histopathological changes. Allele losses were not random and followed a sequence. This study refines several distinct chromosome 3p, 8p, 9q and 22q regions undergoing frequent allele loss in gallbladder carcinoma that will aid in the positional identification of tumour suppressor genes involved in gallbladder carcinoma pathogenesis.

British Journal of Cancer (2002) 87, 432 - 440. doi:10.1038/sj.bjc.6600490 www.bjcancer.com

(C) 2002 Cancer Research UK

Keywords: loss of heterozygosity; tumour suppressor gene; microsatellite marker; preneoplasia; microdissection

Gallbladder carcinoma (GBC) is a relatively uncommon neoplasm which demonstrates considerable geographic and gender variation in incidence (Albores-Saavedra and Henson, 2000). For unknown reasons, it is one of the most frequent neoplasms in Chile, where it is the leading cause of cancer deaths in females (Lazcano-Ponce et $a l, 2001)$. It has been well established that invasive GBC is preceded by preneoplastic lesions, including dysplastic changes of the gallbladder epithelium (Albores-Saavedra and Henson, 2000). There is a very limited information about the molecular changes involved in the pathogenesis of GBC (Wistuba and Albores-Saavedra, 1999).

It is now well recognised that tumourigenesis is a multistep process resulting from the accumulation of sequential genetic alterations (Fearon and Vogelstein, 1990). In addition to oncogene activation, inactivation of tumour suppressor genes (TSGs) has been shown to play an important role in tumourigenesis (Fearon and Vogelstein, 1990). Allelic loss, manifested as loss of heterozygosity (LOH) at polymorphic loci, is recognised as a hallmark of tumour suppressor genes, whose other allele is inactivated by point mutations or by some other mechanism (Knudson, 1985). Thus, the finding of chromosomal regions with frequent incidence of $\mathrm{LOH}$ in a neoplasm suggests that those regions may harbour one or more TSGs.

Our recent genome-wide allelotyping analysis on GBC indicated that allelic losses at multiple sites of the genome are frequent in this neoplasm (Wistuba et al, 2001). We identified at least 21 chromosomal regions with frequent $\mathrm{LOH}$ in GBC, including $3 p, 8 p, 9 q$

*Correspondence: II Wistuba; E-mail: iwistuba@med.puc.cl

Received 2I February 2002; revised 29 April 2002; accepted 7 June 2002 and 22q regions. Allelic losses at those chromosome sites have been implicated in the pathogenesis of several human neoplasms, suggesting that there probably are several different TSGs located in those chromosomal regions (Albrecht et al, 1994; Chaganti et al, 1995; Habuchi et al, 1995; El-Naggar et al, 1998; Miyakawa et al, 1998; Cheng et al, 1999; Wistuba et al, 1999a, 2000; Baffa et al, 2000; Choi et al, 2000; Lerman and Minna, 2000; Muscheck et al, 2000; Maitra et al, 2001).

The present study was undertaken to refine the regions of frequent allele loss involving chromosomes $3 p, 8 p, 9 q$ and $22 q$ in GBC identified with in our recent genome-wide allelotyping analysis (Wistuba et al, 2001). Using DNA extracted from archival paraffin-embedded tissue of GBC, and a panel of high-resolution polymorphic markers, we studied the shortest regions of overlapping $\mathrm{LOH}$ on chromosomes $3 \mathrm{p}, 8 \mathrm{p}, 9 \mathrm{q}$ and $22 \mathrm{q}$. We also studied the role of those allele losses in GBC pathogenesis by examining $\mathrm{LOH}$ on those four chromosomal arms in gallbladder epithelia accompanying invasive GBC.

\section{MATERIALS AND METHODS}

\section{Archival tumour specimens}

Formalin-fixed paraffin-embedded material from 24 surgically resected primary invasive gallbladder carcinomas was obtained from cholecystectomy specimens resected between 1990 and 1998 at the Catholic University Medical School Hospital, Santiago, Chile, as part of an Institutional Review Board approved study. The patients consisted of 19 women and five men ranging in age from 51-85 
years (mean age, 68 years). Six (25\%) were well differentiated, eleven $(46 \%)$ were moderately differentiated, and seven (29\%) were poorly differentiated tubulo-papillary adenocarcinomas. The majority of the tumours were advanced GBCs (20 of 24 cases; $84 \%$ ) with invasion of the gallbladder serosa; the remaining (four of 24 cases; $16 \%$ ) were early GBCs, with invasion of the submucosa (one case; $4 \%$ ) or muscularis propia (three cases; 12\%) of the gallbladder.

\section{Normal epithelium and preneoplastic lesions accompanying gallbladder cancer}

Forty-five histologically discrete foci of non-invasive gallbladder epithelia were identified adjacent to 20 GBCs, each consisting of at least 1000 cells. These included 17 histologically normal epithelia and 28 high-grade dysplasias (Figure 1). The dysplastic lesions were scored using published criteria for their histopathological identification in the gallbladder epithelium (Albores-Saavedra and Henson, 2000).

\section{Microdissection and DNA extraction}

Serial $5 \mu \mathrm{m}$ sections were cut from archival, formalin-fixed, paraffin-embedded tissues. Precise microdissection from archival paraffin-embedded tissues was performed under microscopic visualization using a micromanipulator, as described previously (Figure 1). From multiple serial sections of each case 4000 to 5000 sectioned tumour cells and 1000 gallbladder epithelial cells were microdissected, and DNA extracted as described. Dissected lymphocytes or normal stromal cells from the same slide were used as a source of constitutional DNA. DNA from at least 200 cells were used for each multiplex PCR reaction, as previously described (Wistuba et al, 1998). In order to circumvent the possibility of artifactual LOH occurring in DNA extracted from the relatively small non-tumour foci, fewer microsatellite markers were analysed to ensure comparable numbers of cells being used for the initial amplification reaction in all samples.

\section{Microsatellite DNA markers and PCR- LOH analysis}

To evaluate $\mathrm{LOH}$ on GBC, we used primers flanking 81 microsatellite repeat polymorphisms spanning several $3 \mathrm{p}(n=26), 8 \mathrm{p}(n=14), 9 \mathrm{q}$ $(n=29)$ and 22q $(n=12)$ regions showing high frequencies of LOH in the genome-wide allelotyping analysis on GBC (Wistuba et al, 2001). The microsatellite markers tested are shown in Figures 2-5. The markers were selected from the Genome Database (http:// www.gdb.org/). Subsets of markers $(n=17)$ spanning a total of 12 chromosomal regions frequently deleted in invasive GBC (Table 1) were used for the analysis of gallbladder epithelium $(3 p=6 ; 8 p=4$; $9 q=4$; and $22 q=3$ ). Because we used DNA extracted from archival paraffin-embedded tissues, the amplicon size was restricted to less than 250 bp. A two-round PCR strategy (multiplex PCR followed by uniplex PCR) was utilised to amplify each marker, as described previously (Wistuba et al, 1998). We optimised the PCR conditions to perform 11 multiplex PCRs containing all 81 markers utilised in this study. Each multiplex PCR contained six to eight microsatellite markers. The list of multiplex sets and specific optimization conditions we used are available upon request. A $10^{\circ} \mathrm{C}$ 'touch-down' PCR strategy was used spanning the primers annealing temperature followed by 25 cycles at the optimal annealing temperature. The final product was separated on a $6 \%$ denaturing polyacrylamide gel and subjected to autoradiography. $\mathrm{LOH}$ was scored by visual detection of complete absence of one allele of informative cases (Figures $2-5$ ).

\section{Data analysis}

The data were analysed using a series of Microsoft Visual Basic programmes specifically written for various computations or repe-
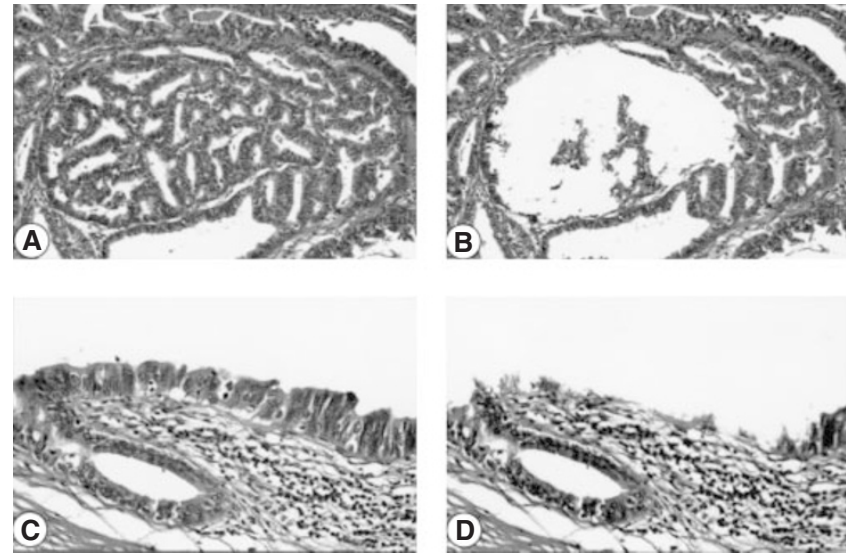

Figure I Representative example of the precise microdissection technique of invasive GBC (A and $\mathbf{B}$ ) and high-grade dysplastic lesion ( $\mathbf{C}$ and $\mathbf{D})$ used in this study. Note that only tumour and epithelial cells were microdissected (A, C, before; $\mathbf{B}, \mathbf{D}$, after) while stromal tissue is intact.

titive tasks in Microsoft Excel, including LOH frequencies, breakpoint frequencies, colour-coded formatting of $\mathrm{LOH}$ patterns, and clustering analysis, as described previously (Girard et al, 2000). In order to determine the sequence of genetic abnormalities in the multistep progression of GBC we utilised multiple microsatellite markers $(n=17)$ to examine $\mathrm{LOH}$ at 12 chromosomal regions located on the four chromosomal arms examined. To determine whether the losses were progressive in the sequential development of GBC, we determined the frequency of loss of individual microsatellite markers $(n=17)$ and chromosomal regions $(n=12)$ using the Fractional Allele Loss (FAL) index and the Fractional Regional Loss (FRL) index, respectively. The FAL index is defined as the total number of microsatellite markers with $\mathrm{LOH}$ in an epithelial sample divided by the total informative markers in the corresponding normal DNA. The FRL index is defined as the total number of chromosomal regions with $\mathrm{LOH}$ divided by the total number of informative regions in the corresponding normal DNA.

Statistical analysis was performed using the nonparametric Wilcoxon and Fisher Exact tests. The cumulative binomial test was used to examine the likelihood that the occurrence of a particular event (loss of the same allele in the invasive carcinoma and an associated epithelial sample) occurs at a particular probability when observed in repeated trials. When the results are compared with a chance occurrence or nonoccurrence, the particular probability of comparison is 0.5 . Probability values of $P<0.05$ were regarded as statistically significant.

\section{RESULTS}

\section{Definition of regions of loss in GBC}

Overall frequencies of allelic loss at any $3 \mathrm{p}$ (24 out of 24 cases, $100 \%), 8 p$ (24 out of 24 cases, 100\%), 9q (21 out of 24 cases, $88 \%$ ), and $22 \mathrm{q}$ ( 22 out of 24 cases, $92 \%$ ) sites were very high in GBCs. Most of the sites of allelic loss at all chromosomal arms examined were localised, and the extent of the partial allele losses was used to identify 13 discrete minimal regions of non-overlapping allele losses (four for $3 \mathrm{p}$; three for $8 \mathrm{p}$; four for $9 \mathrm{q}$; and two for 22q). The patterns of $\mathrm{LOH}$ and frequencies of allelic loss at different critical regions identified are shown in Figures $2-5$ and summarised in Table 2. Representative autoradiographs for cases with partial loss of all four chromosomal arms demonstrating loss and retention of neighbouring alleles in GBCs are illustrated in Figures 2-5. Because artifacts resulting from PCR amplification may be mistaken for $\mathrm{LOH}$, especially when minute amounts of input DNA are utilised, approximately $30 \%$ of examples of $\mathrm{LOH}$, 


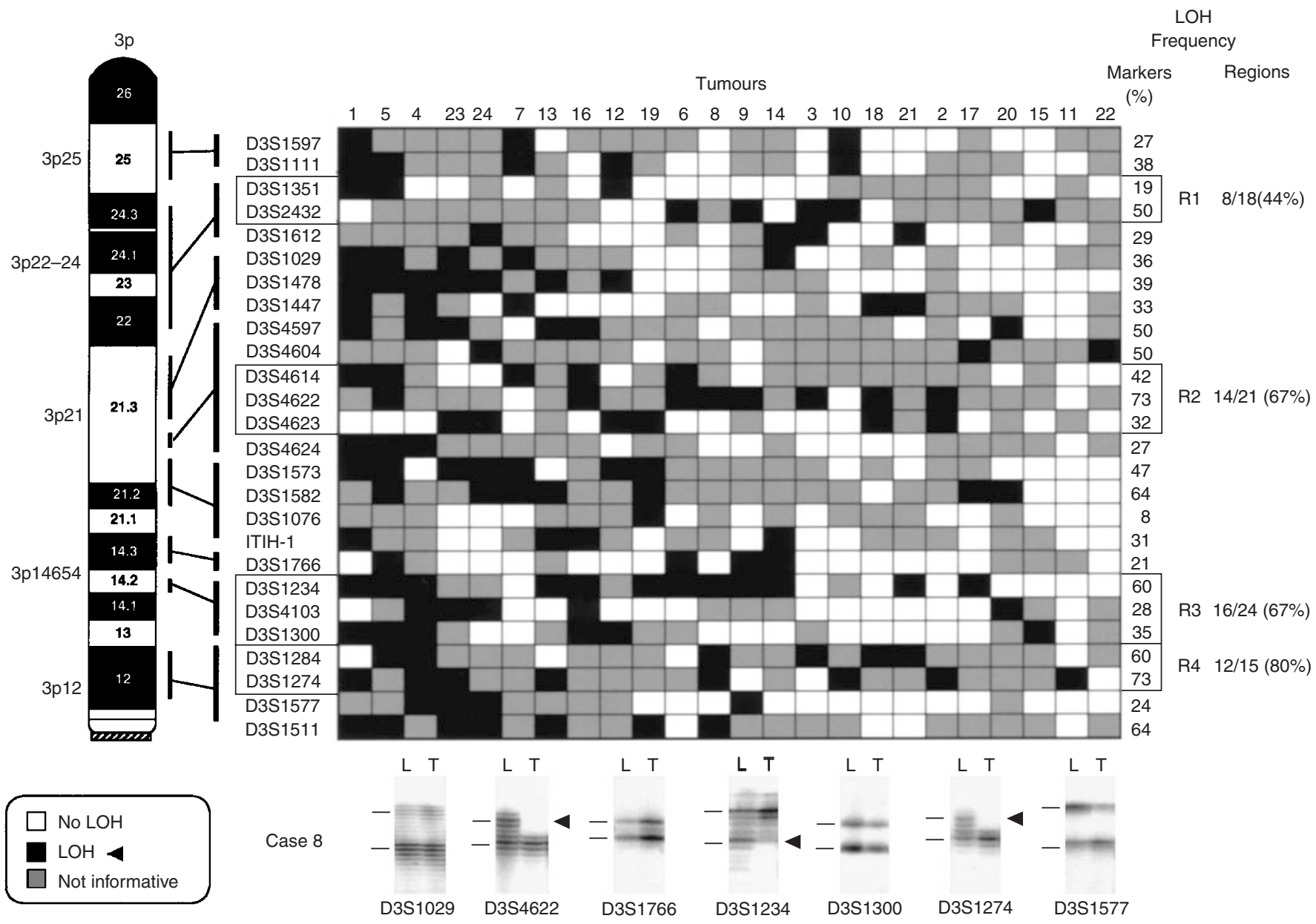

Figure 2 Patterns of chromosome 3p allele losses in gallbladder carcinoma. The cases have been arranged from left to right in decreasing order of chromosome $3 p$ allele losses. Markers are placed in the predicted order from 3pter-cen. Four regions $(R I-R 4)$ having frequent allelic losses are shown. Lower panel: seven autoradiographs showing discrete allele loss at chromosome 3p in a microdissected GBC case (case 8) demonstrating loss and retention of neighbouring alleles. L, lymphocytes or normal stromal cells; T, microdissected invasive GBC. Horizontal bars on the left of the autoradiographs indicate the main allelic bands.

including many foci having distinct losses, were repeated for confirmation from newly microdissected material and identical $\mathrm{LOH}$ pattern were detected.

\section{Patterns of loss in GBC}

Although most of the losses at all chromosomal arms examined were localised, the data demonstrate that in chromosomes $3 \mathrm{p}, 8 \mathrm{p}$ and $9 \mathrm{q}$ the most frequently observed pattern was loss of two or more regions. Thus, allelic loss of a single region in each of those chromosomes by itself was a relatively infrequent event. Two or more regions were lost in $84 \%$ at $3 p, 73 \%$ at $8 p, 63 \%$ at $9 q$, and $52 \%$ at $22 \mathrm{q}$. Cluster analysis to examine if allelic loss at one chromosomal region was linked to changes at another region (Girard et al, 2000) did not reveal any concordance between markers from the same or different chromosomes.

\section{Allelic loss in normal and dysplastic gallbladder epithelia accompanying tumours}

Allelic loss at one or more $3 p, 8 p, 9 q$ and $22 q$ regions was detected in the majority of the histologically normal (15 out of $17,88 \%)$ and dysplastic (26 out of $28,93 \%$ ) foci examined (Table 1). The FAL (Fractional Allelic Loss) and FRL (Fractional Regional Loss) indices were calculated as an expression of the amount of allele loss, and an increasing severity of histological changes was characterised by a significant rise of both index means $(P<0.001$; Table 1$)$.

The pattern of allelic loss was not random, and losses at one or more $3 p(54 \%)$ and $8 p(50 \%)$ regions were the most frequently detected abnormalities in histologically normal epithelium (Table 1). While $9 q$ allelic loss was relatively frequent (29\%) in normal epithelium, losses at $22 \mathrm{q}$ commenced at the dysplasia stage. For most of the chromosomes examined ( $3 p, 8 p$ and $22 q$ ), the differences between dysplasia and invasive carcinoma were modest and not significant. Data on individual epithelial and tumour samples are pooled in Figure 6. As minute amounts of input DNA were utilised, all examples of LOH from the histologically normal epithelia and nearly half from the dysplasia were repeated for confirmation.

\section{Patterns of allelic loss in the pathogenesis of GBC}

To determine the sequential molecular changes involved in the development of GBC, we analysed the pattern of allele losses detected in the GBC tumours and their accompanying normal and dysplastic epithelia. We considered only 14 tumours and 30 accompanying normal $(n=11)$ and dysplastic $(n=19)$ epithelial specimens that were informative for at least one marker in each 

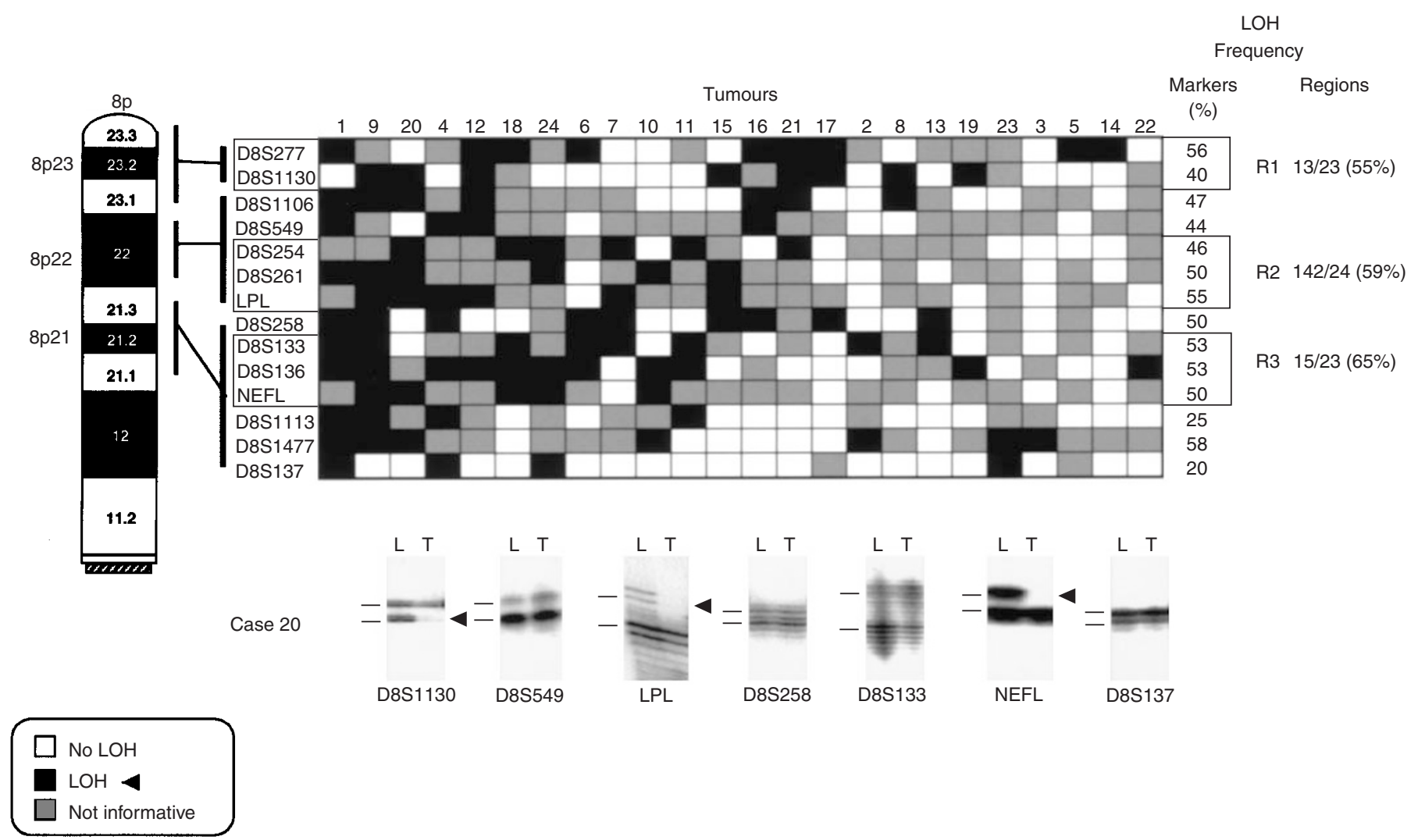

Figure 3 Patterns of chromosome $8 p$ allele losses in gallbladder carcinoma. The cases have been arranged from left to right in decreasing order of chromosome 8p allelic losses. Markers are placed in the predicted order from 8pter-cen. Three regions $(R I-R 3)$ having frequent allelic losses are shown. Lower panel: seven autoradiographs showing discrete allele loss at chromosome 8p in a microdissected GBC case (case 20) demonstrating loss and retention of neighbouring alleles. L, lymphocytes or normal stromal cells; T, microdissected invasive GBC. Horizontal bars on the left of the autoradiographs indicate the main allelic bands.

chromosomal arm examined. From these specimens only a single dysplastic focus showed no $\mathrm{LOH}$ at any chromosomal arm and was excluded from this analysis. Three patterns of allelic loss were discerned in the 30 histologically normal, dysplastic and neoplastic foci: (a) Early pattern, with only $8 \mathrm{p}$ loss or $8 \mathrm{p}$ accompanied with $3 p$. (b) Intermediate pattern, with only $9 q$ loss or $9 q$ accompanied with $3 p$ and/or $8 p$. (c) Advanced pattern, with $22 q$ loss accompanied with $8 p+3 p$ or $8 p+9 q$ or $8 p+3 p+9 q$. Of interest, normal gallbladder epithelium had only early $(55 \%)$ and intermediate (45\%) patterns, while tumours had advanced (71\%) and intermediate $(29 \%)$ patterns. Dysplastic gallbladder epithelia demonstrated the entire spectrum of patterns, including the advanced (56\%), intermediate (30\%) and early (14\%).

\section{Allele specific mutations $v s$ potential clonal relationship of epithelial foci}

Previous studies in gallbladder carcinoma and other neoplasms demonstrated that at any one locus, loss of parental alleles was not random, and that there was a strong tendency for the identical allele to be lost in all non-neoplastic and neoplastic foci examined (Wistuba et al, 1995, 1999b). We refer to this phenomenon as allele specific mutation (ASM) (Wistuba et al, 1995, 1999b). We determined the frequencies of ASM in the 61 epithelial and tumoural foci demonstrating one or more sites of allelic loss in $20 \mathrm{GBC}$ cases. For all 146 comparisons involving 17 microsatellite markers, the same parental allele was lost in $128(88 \%)$. The possibility that this occurred by chance alone is extremely remote as tested by the cumulative binomial test $\left(P=5.3 \times 10^{-22}\right)$.
Because in the GBC there is a close morphological relationship between invasive carcinoma and its dysplastic lesions (AlboresSaavedra and Henson, 2000) we then examined the possibility that cancer and non-malignant gallbladder epithelia were clonally related, using the specific alleles lost to mark the different foci in individual cases. Possible evidence of a clonal relationship (through sequential changes) were present only in $10(16 \%)$ out of 61 foci examined. In most $(84 \%)$ of the normal and dysplastic epithelia the patterns of allele loss suggested that they arose as independent clones. However, we cannot exclude the possibility that the lesions represent subclones from a precursor clone having some initial molecular change, which we did not examine. Despite this lack of clonal relationship ASM was still significantly detected even in the analysis of clonally unrelated foci (104 of 123 comparisons, $\left.85 \% ; P=9.1 \times 10^{-26}\right)$.

\section{Microsatellite instability (MSI) in GBC pathogenesis}

We detected a relatively high frequency of MSI at one or more $3 p$, $8 \mathrm{p}, 9 \mathrm{q}$ and $22 \mathrm{q}$ chromosomal loci in one of $13(8 \%)$ of histologically normal foci, in four of $54(7 \%)$ dysplastic foci and in six of 12 $(50 \%)$ invasive carcinomas examined (data not shown).

\section{DISCUSSION}

Our recent genome-wide allelotyping analysis indicated that allelic losses at multiple sites of the genome are frequent in $\mathrm{GBC}$, and indicated for the first time that in addition to $3 p$ and $8 \mathrm{p}, \mathrm{LOH}$ on $9 \mathrm{q}$ and 22q may also play a role in the pathogenesis of this neoplasm 


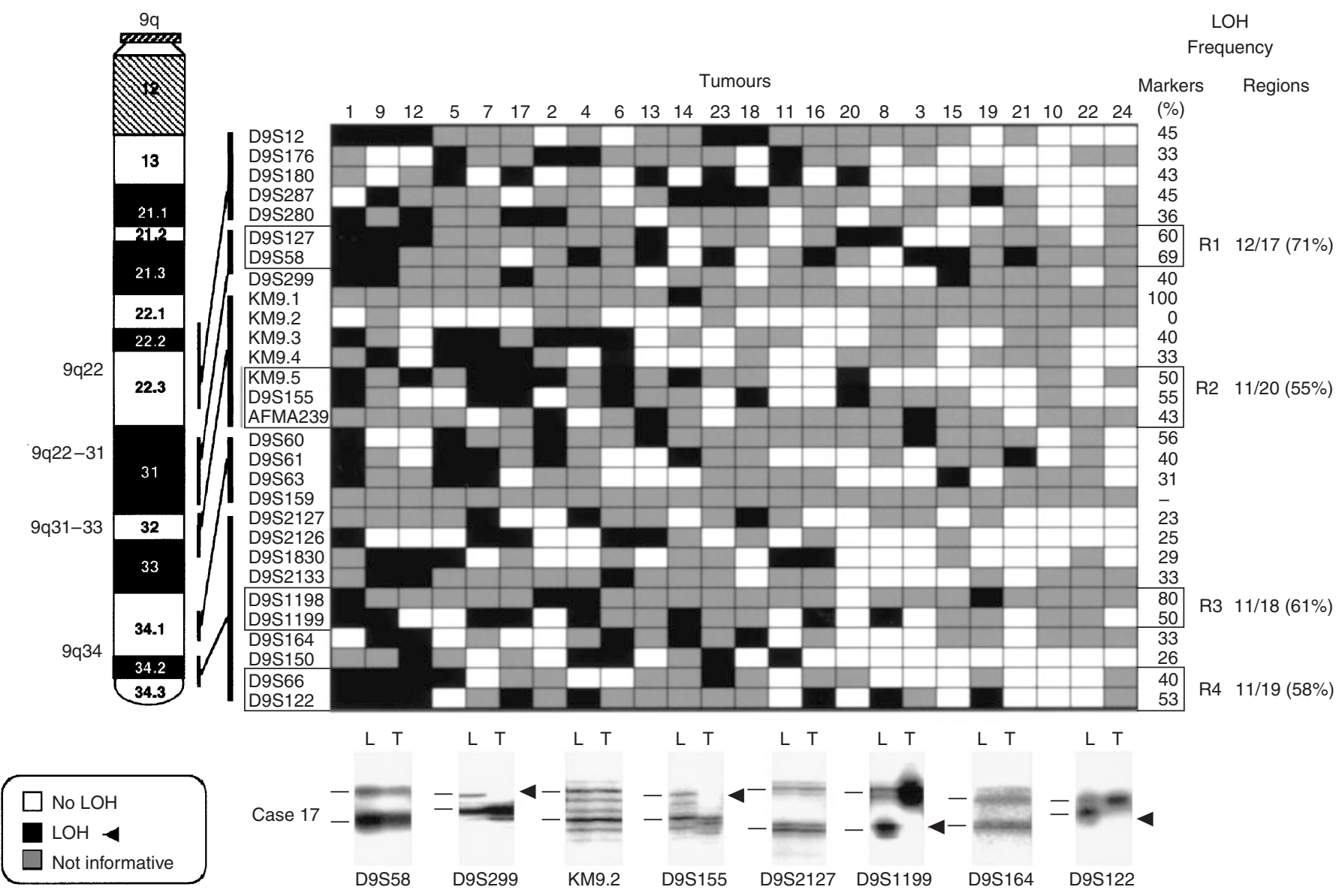

Figure 4 Patterns of chromosome $9 \mathrm{q}$ allele losses in gallbladder carcinoma. The cases have been arranged from left to right in decreasing order of chromosome $9 q$ allele losses. Markers are placed in the predicted order from 9qcen-ter. Four regions $(R /-R 4)$ having frequent allelic losses are shown. Lower panel: eight autoradiographs showing discrete allele loss at chromosome $9 \mathrm{q}$ in a microdissected GBC case (case 17) demonstrating loss and retention of neighbouring alleles. L, lymphocytes or normal stromal cells; T, microdissected invasive GBC. Horizontal bars on the left of the autoradiographs indicate the main allelic bands.

(Wistuba et al, 2001). We identified at least 21 chromosomal regions with frequent $\mathrm{LOH}$ in GBC, including sites on $3 \mathrm{p}, 8 \mathrm{p}, 9 \mathrm{q}$ and $22 \mathrm{q}$, suggesting that those chromosome regions may harbour putative TSGs that are inactivated in the development of this tumour. This previous study was a low-density screen in order to elucidate chromosomal hot spots for subsequent detailed analysis.

The present study with high-density allelotyping have confirmed and extended the previous findings. The overall frequencies of allelic loss at any $3 p(100 \%), 8 p(100 \%), 9 q(88 \%)$, and $22 q(92 \%)$ sites were very high in our tumours. Overall, thirteen distinct sites of frequent allele loss in GBC at chromosome 3p, 8p, 9q and 22q (summarised in Table 2) were detected in the present study, and the majority of these have not previously been described in this neoplasm. The location of these sites was based on the determination of the minimal region of loss that is defined by the occurrence of 'breakpoints' surrounding regions of frequent LOH. These sites are likely to represent TSG regions that are lost in GBC and warrant further investigation.

As a result of our detailed allelotyping $3 p$ analysis on GBC, we were able to identify multiple areas of discontinuous $\mathrm{LOH}$ and four distinct $3 p$ regions $(3 \mathrm{p} 22-24 ; 3 \mathrm{p} 21.3$; $3 \mathrm{p} 14.2$; and $3 \mathrm{p} 12)$ with frequent allelic loss in this tumour. Several candidate TSGs have been detected in those $3 p$ regions with frequent allele losses in GBC. One candidate in the 3p22-24 region is the retinoic acid receptor-beta $(R A R \beta)$ gene (Virmani et al, 2000). Another candidate gene, the fragile histidine triad (FHIT) gene, spans the FRA3B fragile site at 3p14.2 (Huebner et al, 1998). A new candi- date TSG, ROB01 (DUTT1), has been cloned residing in the U2020 3p12 deletion region at marker D3S1274 (Sundaresan et al, 1998). Currently two distinct $3 \mathrm{p} 21.3$ regions are under study because of the existence of multiple homozygous allele loss in lung and breast cancer cell lines (Lerman and Minna, 2000). One of those was tested in this report by markers D3S4614 and D354622, and represents a minimal region of frequent allele losses $(67 \%)$ in our GBC cases. Recently, a new candidate TSG located in this region, the human RAS effector homologue (termed RASSF1A) gene, has been shown to have tumour suppressing function and undergoes epigenetic inactivation in several cancers (Dammann et al, 2000; Burbee et al, 2001; Dreijerink et al, 2001).

Our detailed allelotyping analysis of the 8p21-23 region demonstrated three distinct regions with frequent allelic loss on GBC, namely $8 \mathrm{p} 23,8 \mathrm{p} 22$, and $8 \mathrm{p} 21$. The $8 \mathrm{p} 21$ region, defined by the same markers tested in our GBCs, has been described with frequent allele loss in other tumour types (Trapman et al, 1994; Farrington et al, 1996; Baffa et al, 2000). The platelet-derived growth factor receptor-like gene (PRLTS) candidate TSG is centromeric to our 8p21 minimal region (Fuijwara et al, 1995). The 8p22 region identified in our GBCs spans the FEZ1/LZTS1 gene, a candidate TSG, whose expression is altered in multiple human tumours (Ishii et al, 1999; Cabeza-Arvelaiz et al, 2001). The N33 gene at 8 p22 was found to be silenced in several cancer cells, although no point mutations have been identified (Bookstein $e t$ $a l, 1997)$. Allele losses at the telomeric 8 p23 region, shown to be frequent in our gallbladder tumours, has been previously described 


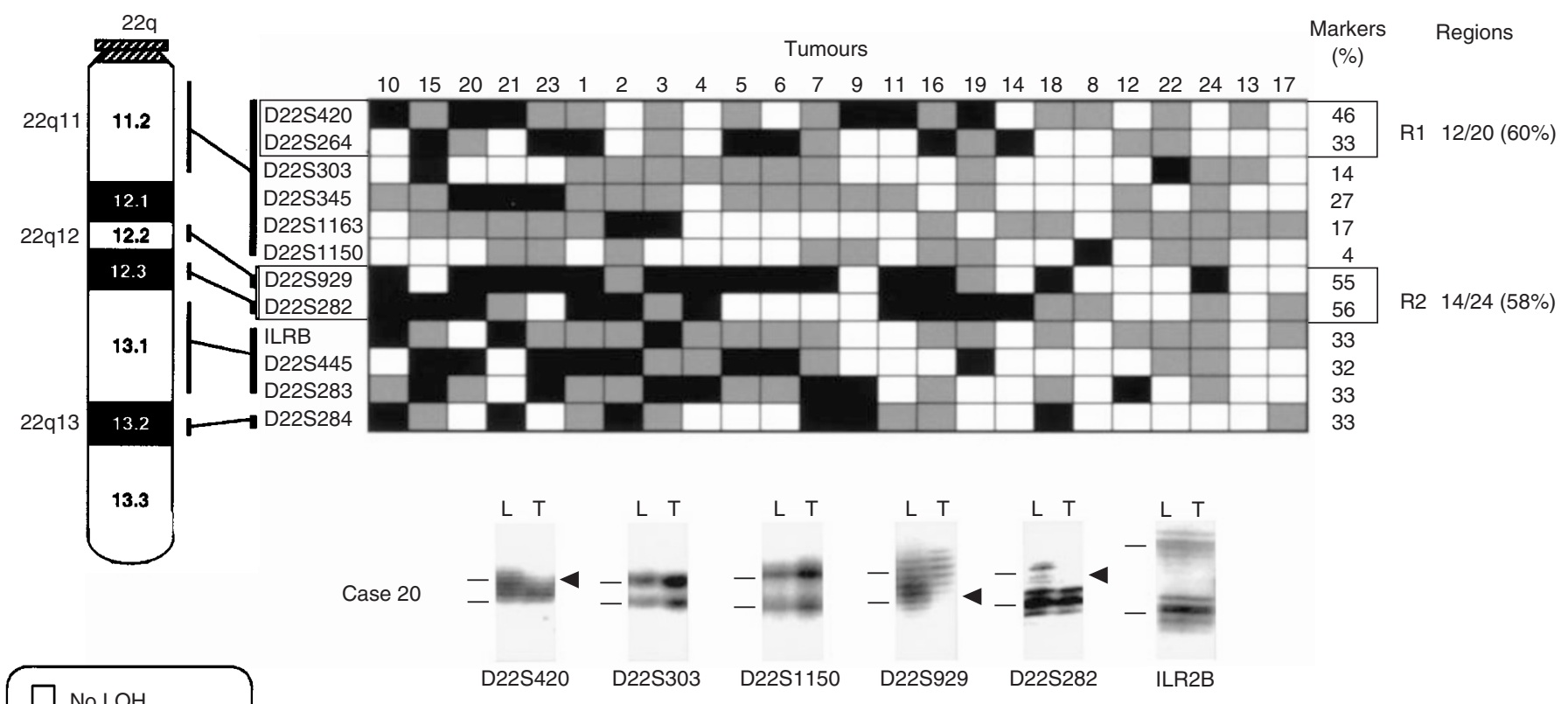

Not informative

Figure 5 Patterns of chromosome 22q allele losses in gallbladder carcinoma. The cases have been arranged from left to right in decreasing order of chromosome 22q allele losses. Markers are placed in the predicted order from 22qcen-ter. Two regions (RI - R2) having frequent allelic losses are shown. Lower panel: six autoradiographs showing discrete allele loss at chromosome 22q in a microdissected GBC case (case 20) demonstrating loss and retention of neighbouring alleles. L, lymphocytes or normal stromal cells; T, microdissected invasive GBC. Horizontal bars on the left of the autoradiographs indicate the main allelic bands.

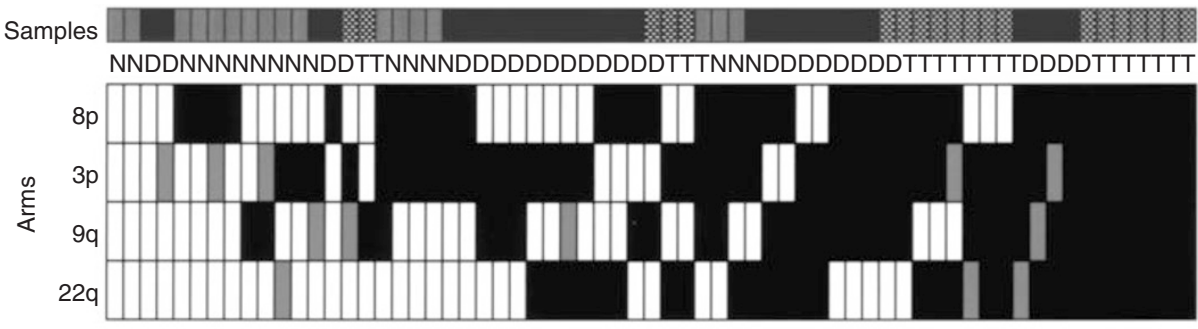

\begin{tabular}{|ll|}
\hline Samples & Chromosomal arms \\
Tormal $(\mathrm{N})$ & $\square \mathrm{No} \mathrm{LOH}$ \\
Dysplasia $(\mathrm{D})$ & $\mathrm{LOH}$ \\
Tumour $(\mathrm{T})$ & $\square$ Not informative \\
\hline
\end{tabular}

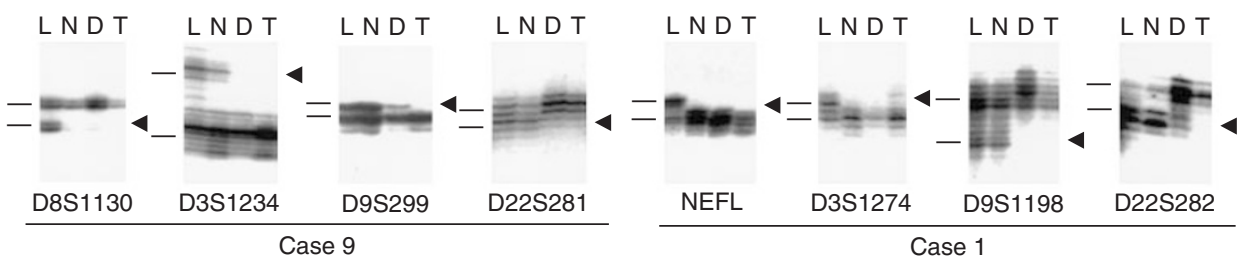

Figure 6 Summary of all allelotyping results by chromosome arms in the pathogenesis of GBC. A total of 69 specimens are shown, including all 24 invasive carcinomas and all 45 non-malignant epithelium ( 17 histologically normal appearing and 28 dysplastic epithelia) accompanying GBCs. The specimens have been sorted from left to right in ascending number chromosome arm allele losses. The sources of the various specimens are coded above the boxes and include normal epithelium $(N)$, dysplasia $(D)$ and tumour $(T)$. Lower panel: eight autoradiographs showing discrete allele loss at chromosomes 3p, 8p, 9q y $22 q$ in two GBCs and their accompanying non-malignant epithelium. L, lymphocytes or normal stromal cells; $N$, normal epithelium; $D$, dysplasia; $T$, microdissected invasive GBC. Horizontal bars on the left of the autoradiographs indicate the main allelic bands. Data analysis shows that allelic losses present in normal and dysplastic epithelia were not random. Analysis of informative samples for all four chromosome arms shows that most normal epithelia demonstrate $8 p$ or $8 p+3 p$ losses and the majority of dysplasias have losses on $8 p$ and/or $3 p$ with $9 q$ and/or 22q, sugesting a sequential model of genetic abnormalities that begins with $8 p \mathrm{LOH}$ and progresses through $3 p, 9 q$ and $22 q$ losses. 
Table I Summary of allelic loss at 3p, 8p, 9q and 22q arms using 17 microsatellite marker loci on GBC and accompanying dysplastic and histologically normal epithelial foci

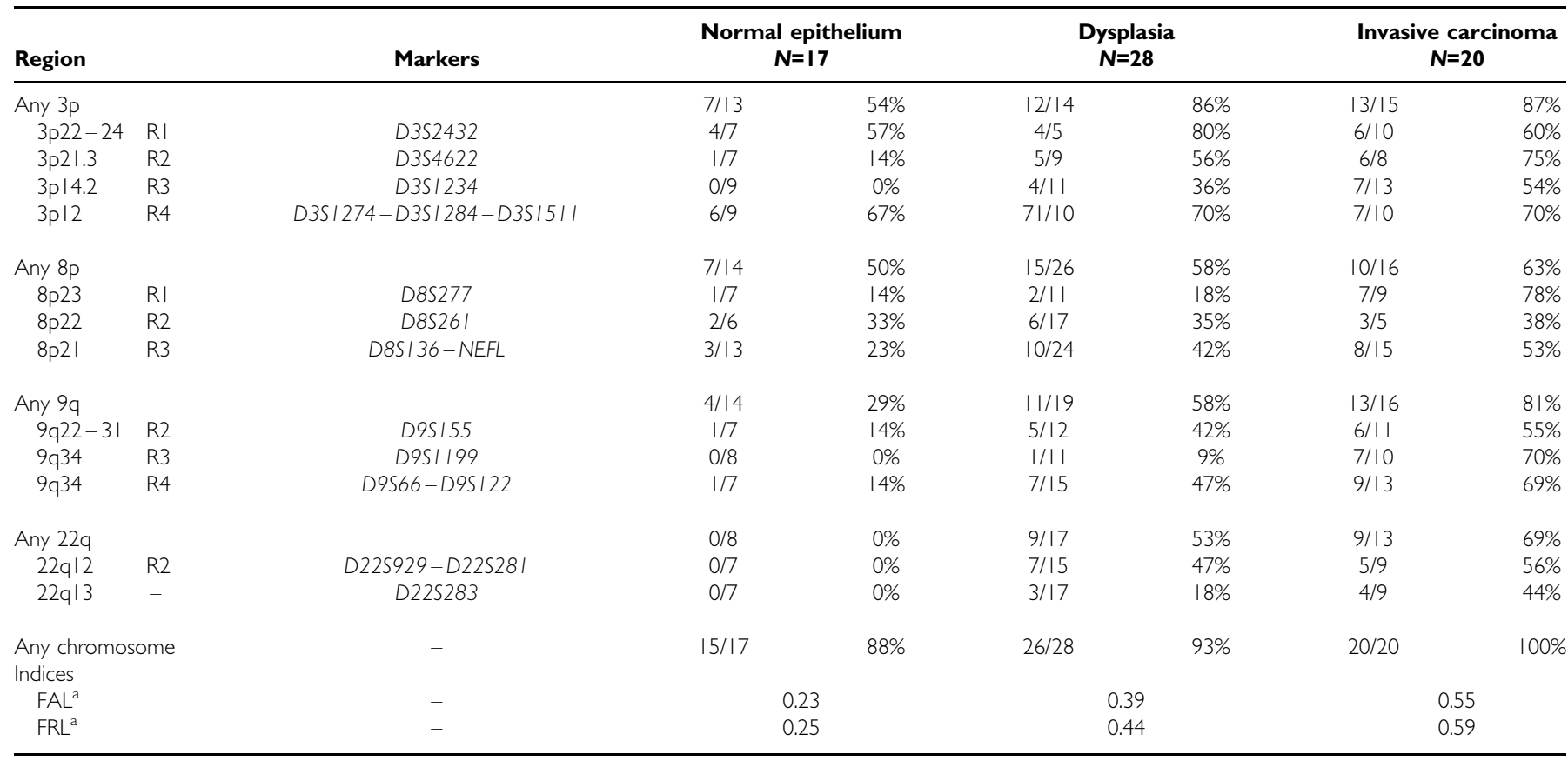

${ }^{\mathrm{a} F A L=F r a c t i o n a l ~ A l l e l e ~ L o s s ; ~}{ }^{\mathrm{b}} \mathrm{FRL}=$ Fractional Regional Loss.

Table 2 Summary of allelic loss at 13 chromosomal regions on 3p, 8p, 9q and 22q examined in gallbladder carcinoma ( $n=24)$

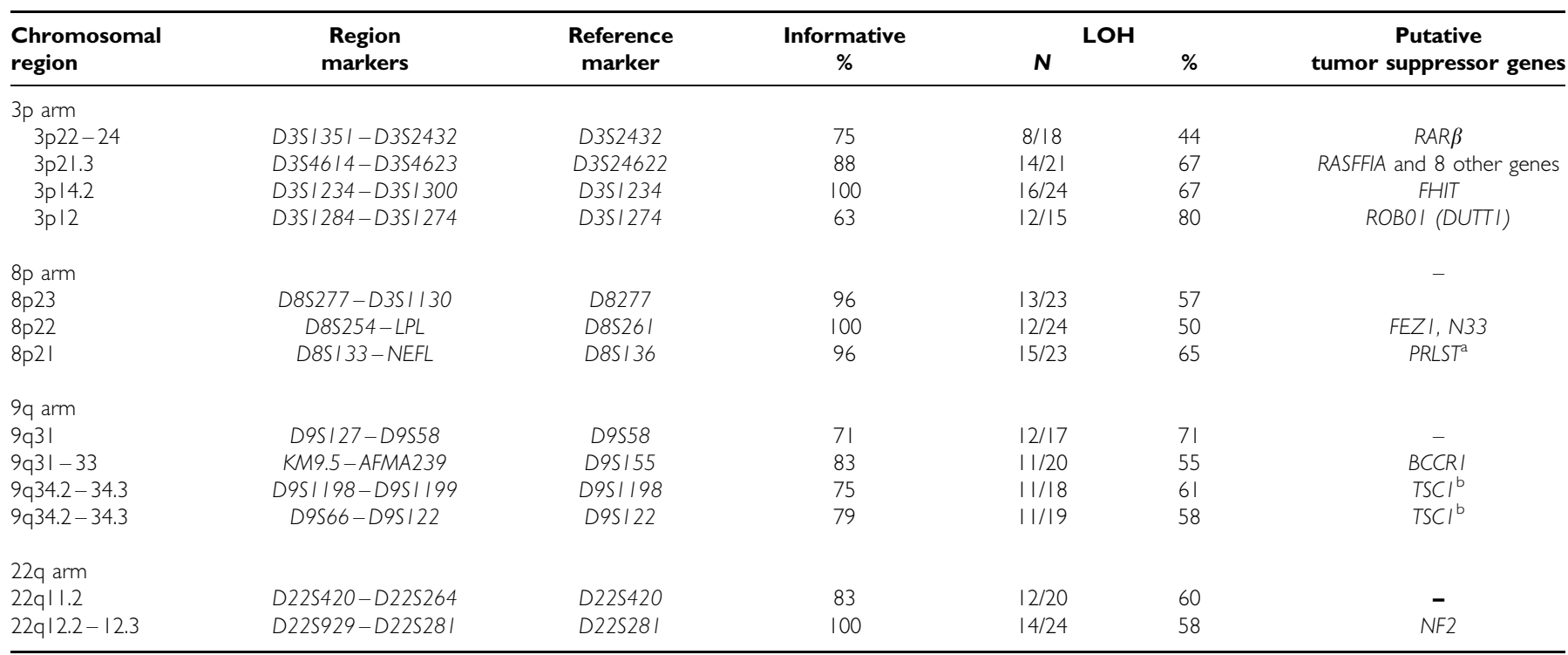

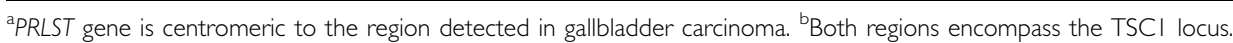

on head and neck, hepatocellular and prostate carcinomas (Perinchery et al, 1999; Pineau et al, 1999; Ishwad et al, 1999). However, no candidate genes have been reported on this region.

At $9 \mathrm{q}$, four different regions of frequent allele losses in GBC were detected, namely $9 \mathrm{q} 31,9 \mathrm{q} 31-33$, and two sites at $9 \mathrm{q} 34.2$ 34.3. While the $9 \mathrm{q} 31$ minimal region has not been previously described in other tumour types, allele losses at the 9q32-33 and $9 q 34$ regions have been frequently reported in other neoplasms (Chaganti et al, 1995). The 9q32-33 minimal region has been intensively studied in transitional cell carcinoma of the urinary bladder (Habuchi et al, 1998, 2001), being designated as DBC1 locus (for deleted in bladder cancer gene 1). A novel candidate gene for this region has been identified and designated as DBCCR1 (Habuchi et al, 1998, 2001). At least two regions of minimal allele losses involving the telomeric 9q34.2-34.3 region were detected in GBC. These regions encompass one of the loci identified for the tuberous sclerosis gene (TSC) (van Slegtenhorst et al, 1997).

We have detected two distinct sites of frequent allelic loss in GBC, 22q11.2 and 22qq12.2-q12.3 regions. Both regions have been described with frequent allele losses in other neoplasms. The centromeric 22q11.2 region has been previously reported in hepatocellular (Takahashi et al, 1993) and breast carcinomas (Allione et al, 1998); 
however, no candidate TSG has been reported at this site. The $22 \mathrm{q} 12.2-\mathrm{q} 12.3$ region encompasses the neurofibromatosis type 2 (NF2) gene, a candidate TSG at this region (Rouleau et al, 1993). Because NF2 gene mutations are rarely seen in epithelial tumours with high frequency of allele losses at the NF2 gene locus (Takahashi et al, 1993; Englefield et al, 1994; Allione et al, 1998; Miyakawa et al, 1998), an additional TSG involved in tumourigenesis could be located at this region.

In several neoplasms it has been established that multiple sequential genetic changes are associated with the development of invasive tumours. However, few investigators have focused in the genetic abnormalities involved in the development of gallbladder carcinoma. We and others (Wistuba et al, 1995; Chang et al, 1999) have reported a high incidence of allelic loss at several chromosomal regions $(17 \mathrm{p}$ TP53, 9p21-p16/CDKN2, and 5q22-APC/MCC region) occurring early during the sequential pathogenesis of GBC. The present findings of frequent chromosome $3 p, 8 p, 9 q$ and $22 q$ allele losses in nonmalignant gallbladder epithelia confirm and greatly extend the findings that molecular changes commence early (in histologically normal epithelium) during the sequential pathogenesis of GBC. Our major findings regarding the molecular pathogenesis of GBC are: (1) molecular changes preceded the onset of histologically recognisable changes and $88 \%$ of the normal histologically normal foci have allele loss at one or more chromosomal regions examined. (2) There was a progressive increase of the overall LOH frequency expressed by the FAL and FRL indices, with increasing severity of histopathological changes. The development of epithelial cancers requires multiple mutations (Fisher, 1958), and the stepwise accumulation of these mutations may represent an inherent mutator phenotype (Loeb, 1991). Thus, it is likely that those preneoplastic lesions that have accumulated multiple mutations are also the ones at higher risk for progression to invasive cancer. (3) Allelic losses present in normal and dysplastic epithelia were not random. The most frequent regions of allelic loss at normal epithelium occurred at $3 \mathrm{p}$ and $8 \mathrm{p}$. While $9 \mathrm{q}$ allelic losses were present mainly in dysplastic lesions, losses at 22q were only detected in advanced lesions (dysplasia and invasive carcinoma). By examining all our material for $3 p, 8 p$, $9 \mathrm{q}$ and $22 \mathrm{q}$ allele loss, we propose a sequential model of genetic abnormalities that begins with $8 \mathrm{p} \mathrm{LOH}$ and progresses through $3 \mathrm{p}$, $9 q$ and 22q. 4) The same parental allele was frequently lost $(88 \%$ of comparisons) in non neoplastic lesions as in the corresponding invasive carcinomas. We refer to this phenomenon as allele specific mutation (ASM). We have documented this phenomenon in several neoplasms (Wistuba et al, 2000; Maitra et al, 2001), including GBC

\section{REFERENCES}

Albores-Saavedra J, Henson DE (2000) Tumours of gallbladder and extrahepatic bile ducts Fascicle 23, 3rd edn, Washington, DC: Armed Forces Institute of Pathology

Albrecht S, von Deimling A, Pietsch T, Giangaspero F, Brandner S, Kleihues P, Wiestler OD (1994) Microsatellite analysis of loss of heterozygosity on chromosomes 9q, 11p and 17p in medulloblastomas. Neuropathol Appl Neurobiol 20: $74-81$

Allione F, Eisinger F, Parc P, Noguchi T, Sobol H, Birnbaum D (1998) Loss of heterozygosity at loci from chromosome arm 22Q in human sporadic breast carcinomas. Int J Cancer 75: $181-186$

Baffa R, Santoro R, Bullrich F, Mandes B, Ishii H, Croce CM (2000) Definition and refinement of chromosome $8 \mathrm{p}$ regions of loss of heterozygosity in gastric cancer. Clin Cancer Res 6: $1372-1377$

Bookstein R, Bova GS, MacGrogan D, Levy A, Isaacs WB (1997) Tumoursuppressor genes in prostatic oncogenesis: a positional approach. Br J Urol 79(Suppl 1): $28-36$

Burbee DG, Forgacs E, Zochbauer-Muller S, Shivakumar L, Fong K, Gao B, Randle D, Kondo M, Virmani A, Bader S, Sekido Y, Latif F, Milchgrub S, Toyooka S, Gazdar AF, Lerman MI, Zabarovsky E, White M, Minna JD (2001) Epigenetic inactivation of RASSF1A in lung and breast cancers and malignant phenotype suppression. J Natl Cancer Inst 93: 691 -699
(Wistuba et al, 1995). The mechanism underlying ASM remains unknown. Possibilities include the following: (a) seeding of multiples sites in the gallbladder epithelium with a common progenitor clone; (b) inhereted differences (e.g., polymorphism) in alleles that determine their susceptibility to loss; and, (c) potential epigenetic differences in alleles that arise during development (e.g., methylation) that might predispose one to preferential loss. Since there is a close morphological relationship between invasive carcinoma and dysplastic lesions (Albores-Saavedra and Henson, 2000), we examined the possibility that the ASM phenomenon represented clonal relationship due to a seeding or spread in the gallbladder epithelium from a common progenitor clone. However, our findings that most $(84 \%)$ of the normal and dysplastic gallbladder epithelia accompanying invasive tumours arose as independent clones suggest that ASM has a different basis than clonality in GBC, such as inhereted susceptibility to loss or potential epigenetic differences that might predispose to preferential allele loss.

Alterations in microsatellite size, microsatellite instability (MSI), are present in many cancers, including GBC (Wistuba et al, 1995), and reflect a form of genetic instability. We found MSI in 50\% of the invasive GBC tumours and in lesser percentages of histologically normal and dysplastic foci. The mechanism involving MSI in GBC need to be further studied.

In summary, allele losses of chromosome $3 p, 8 p, 9 q$ and $22 q$ regions are frequent in GBC. Our data identifies 13 distinct regions of loss on those chromosomal arms, many of which harbour one or more candidate TSGs, which may play a role in GBC pathogenesis. These regions have previously been reported to be frequently lost in several human cancers, suggesting that may harbour TSGs whose inactivation may be critical to the process of tumourigenesis. In addition, our findings in the non-malignant gallbladder epithelium indicate that multiple, non-random and sequential allele specific abnormalities commence early in the multistage pathogenesis of GBC. These findings should be useful for the identification of the TSGs involved in the pathogenesis of GBC, with the potential for defining molecular markers for early detection as well as for the development of gene therapy strategies of this highly malignant neoplasm.

\section{ACKNOWLEDGEMENTS}

Supported by grant FONDECYT (Fondo Nacional de Desarrollo Cientifico y Tecnologico) 1990489 (II Wistuba), the G Harold and Leila Y Mathers Charitable Foundation, and CA71618 (JD Minna)

Cabeza-Arvelaiz Y, Sepulveda JL, Lebovitz RM, Thompson TC, Chinault AC (2001) Functional identification of LZTS1 as a candidate prostate tumour suppressor gene on human chromosome 8p22. Oncogene 20: 4169-4179

Chaganti SR, Gaidano G, Louie DC, Dalla-Favera R, Chaganti RS (1995) Diffuse large cell lymphomas exhibit frequent deletions in 9p21-22 and 9q31-34 regions. Genes Chromosomes Cancer 12: $32-36$

Chang HJ, Kim SW, Kim YT, Kim WH (1999) Loss of heterozygosity in dysplasia and carcinoma of the gallbladder. Mod Pathol 12: $763-769$

Cheng JQ, Lee WC, Klein MA, Cheng GZ, Jhanwar SC, Testa JR (1999) Frequent mutations of NF2 and allelic loss from chromosome band 22q12 in malignant mesothelioma: evidence for a two-hit mechanism of NF2 inactivation. Genes Chromosomes Cancer 24: 238-242

Choi C, Kim MH, Juhng SW, Oh BR (2000) Loss of heterozygosity at chromosome segments $8 \mathrm{p} 22$ and $8 \mathrm{p} 11.2-21.1$ in transitional-cell carcinoma of the urinary bladder. Int J Cancer 86: 501-505

Dammann R, Li C, Yoon JH, Chin PL, Bates S, Pfeifer GP (2000) Epigenetic inactivation of a RAS association domain family protein from the lung tumour suppressor locus 3p21.3. Nat Genet 25: 315-319 
Dreijerink K, Braga E, Kuzmin I, Geil L, Duh FM, Angeloni D, Zbar B, Lerman MI, Stanbridge EJ, Minna JD, Protopopov A, Li J, Kashuba V, Klein G, Zabarovsky ER (2001) The candidate tumour suppressor gene, RASSF1A, from human chromosome 3 p21.3 is involved in kidney tumourigenesis. Proc Natl Acad Sci USA 98: 7504-7509

El-Naggar AK, Coombes MM, Batsakis JG, Hong WK, Goepfert H, Kagan J (1998) Localization of chromosome $8 p$ regions involved in early tumourigenesis of oral and laryngeal squamous carcinoma. Oncogene 16: 2983 2987

Englefield P, Foulkes WD, Campbell IG (1994) Loss of heterozygosity on chromosome 22 in ovarian carcinoma is distal to and is not accompanied by mutations in NF2 at 22q12. Br J Cancer 70: 905-907

Farrington SM, Cunningham C, Boyle SM, Wyllie AH, Dunlop MG (1996) Detailed physical and deletion mapping of $8 \mathrm{p}$ with isolation of YAC clones from tumour suppressor loci involved in colorectal cancer. Oncogene 12: $1803-1808$

Fearon ER, Vogelstein B (1990) A genetic model for colorectal tumourigenesis. Cell 61: 759-767

Fisher JC (1958) Multiple mutation theory of carcinogenesis. Nature 181: $651-652$

Fujiwara Y, Ohata H, Kuroki T, Koyama K, Tsuchiya E, Monden M, Nakamura Y (1995) Isolation of a candidate tumour suppressor gene on chromosome 8p21.3-p22 that is homologous to an extracellular domain of the PDGF receptor beta gene. Oncogene 10: 891-895

Girard L, Zochbauer-Muller S, Virmani AK, Gazdar AF, Minna JD (2000) Genome-wide allelotyping of lung cancer identifies new regions of allelic loss, differences between small cell lung cancer and non-small cell lung cancer, and loci clustering. Cancer Res 60: 4894-4906

Habuchi T, Devlin J, Elder PA, Knowles MA (1995) Detailed deletion mapping of chromosome $9 \mathrm{q}$ in bladder cancer: evidence for two tumour suppressor loci. Oncogene 11: 1671-1674

Habuchi T, Luscombe M, Elder PA, Knowles MA (1998) Structure and methylation-based silencing of a gene (DBCCR1) within a candidate bladder cancer tumour suppressor region at 9q32-q33. Genomics 48: 277-288

Habuchi T, Takahashi T, Kakinuma H, Wang L, Tsuchiya N, Satoh S, Akao T, Sato K, Ogawa O, Knowles MA, Kato T (2001) Hypermethylation at 9q32-33 tumour suppressor region is age-related in normal urothelium and an early and frequent alteration in bladder cancer. Oncogene 20: $531-537$

Huebner K, Garrison PN, Barnes LD, Croce CM (1998) The role of the FHIT/ FRA3B locus in cancer. Annu Rev Genet 32: 7-31

Ishii H, Baffa R, Numata SI, Murakumo Y, Rattan S, Inoue H, Mori M, Fidanza V, Alder H, Croce CM (1999) The FEZ1 gene at chromosome 8 p22 encodes a leucine-zipper protein, and its expression is altered in multiple human tumours. Proc Natl Acad Sci USA 96: 3928-3933

Ishwad CS, Shuster M, Bockmuhl U, Thakker N, Shah P, Toomes C, Dixon M, Ferrell RE, Gollin SM (1999) Frequent allelic loss and homozygous deletion in chromosome band 8p23 in oral cancer. Int J Cancer 80: 25-31

Knudson AG (1985) Hereditary cancer, oncogenes, and antioncogenes. Cancer Res 45: 1437 - 1443

Lazcano-Ponce E, Miquel JF, Ferrecio C, Wistuba I, Muñoz N, Alonso de Ruiz P, Urista G, Herrero R, Nervi F (2001) Epidemiology and Molecular Pathology of Gallbladder Cancer. Ca Cancer J Clin 51: 349-364

Lerman MI, Minna JD (2000) The 630-kb lung cancer homozygous deletion region on human chromosome 3 p21.3: identification and evaluation of the resident candidate tumour suppressor genes. The International Lung Cancer Chromosome 3p21.3 Tumour Suppressor Gene Consortium. Cancer Res 60: 6116-6133

Loeb LA (1991) Mutator phenotype may be required for multistage carcinogenesis. Cancer Res 51: 3075-3079

Maitra A, Wistuba II, Washington C, Virmani AK, Ashfaq R, Milchgrub S, Gazdar AF, Minna JD (2001) High-resolution chromosome 3p allelotyping of breast carcinomas and precursor lesions demonstrates frequent loss of heterozygosity and a discontinuous pattern of allele loss. Am J Pathol 159: $119-130$

Miyakawa A, Wang XL, Nakanishi H, Imai FL, Shiiba M, Miya T, Imai Y, Tanzawa H (1998) Allelic loss on chromosome 22 in oral cancer: possibility of the existence of a tumour suppressor gene on 22q13. Int J Oncol 13: $705-709$
Muscheck M, Sukosd F, Pesti T, Kovacs G (2000) High density deletion mapping of bladder cancer localizes the putative tumour suppressor gene between loci D8S504 and D8S264 at chromosome 8p23.3. Lab Invest 80: $1089-1093$

Perinchery G, Bukurov N, Nakajima K, Chang J, Hooda M, Oh BR, Dahiya R (1999) Loss of two new loci on chromosome 8 (8p23 and 8q12-13) in human prostate cancer. Int J Oncol 14: 495-500

Pineau P, Nagai H, Prigent S, Wei Y, Gyapay G, Weissenbach J, Tiollais P, Buendia MA, Dejean A (1999) Identification of three distinct regions of allelic deletions on the short arm of chromosome 8 in hepatocellular carcinoma. Oncogene 18: $3127-3134$

Rouleau GA, Merel P, Lutchman M, Sanson M, Zucman J, Marineau C, Hoang-Xuan K, Demczuk S, Desmaze C, Plougastel B, Pulst SM, Lenoir G, Bijisma E, Fashold R, Dumanski J, de Jong P, Parry D, Eldrige R, Aurias A, Delattre O, Thomas G (1993) Alteration in a new gene encoding a putative membrane-organizing protein causes neuro-fibromatosis type 2. Nature 363: 515-521

Sundaresan V, Chung G, Heppell-Parton A, Xiong J, Grundy C, Roberts I, James L, Cahn A, Bench A, Douglas J, Minna J, Sekido Y, Lerman M, Latif F, Bergh J, Li H, Lowe N, Ogilvie D, Rabbitts P (1998) Homozygous deletions at 3p12 in breast and lung cancer. Oncogene 17: 1723-1729

Takahashi K, Kudo J, Ishibashi H, Hirata Y, Niho Y (1993) Frequent loss of heterozygosity on chromosome 22 in hepatocellular carcinoma. Hepatology 17: $794-799$

Trapman J, Sleddens HF, van der Weiden MM, Dinjens WN, Konig JJ, Schroder FH, Faber PW, Bosman FT (1994) Loss of heterozygosity of chromosome 8 microsatellite loci implicates a candidate tumour suppressor gene between the loci D8S87 and D8S133 in human prostate cancer. Cancer Res 54: 6061 - 6064

van Slegtenhorst M, de Hoogt R, Hermans C, Nellist M, Janssen B, Verhoef S, Lindhout D, van den Ouweland A, Halley D, Young J, Burley M, Jeremiah S, Woodward K, Nahmias J, Fox M, Ekong R, Osborne J, Wolfe J, Povey S, Snell RG, Cheadle JP, Jones AC, Tachataki M, Ravine D, Sampson JR, Reeve MP, Richardson P, Wilmer F, Munro C, Hawkins TL, Sepp T, Ali JB, Ward S, Green AJ, Yates JR, Kwiatkowski J, Henske EP, Short MP, Haines JH, Jozwiak S, Kwiatkowski DJ (1997) Identification of the tuberous sclerosis gene TSC1 on chromosome 9q34. Science 277: 805-808

Virmani AK, Rahti A, Zöchbauer-Müller S, Sacchi N, Fukuyama Y, Bryant D, Maitra A, Heda S, Fong KM, Thunnissen F, Minna JD, Gazdar AF (2000) Promoter methylation and silencing of the retinoic acid receptor beta gene in lung carcinomas. J Natl Cancer Inst 92: 1303-1307

Wistuba II, Albores-Saavedra J (1999) Genetic abnormalities involved in the pathogenesis of gallbladder carcinoma. J Hepatobiliary Pancreat Surg 6: 237-244

Wistuba II, Behrens C, Milchgrub S, Virmani AK, Jagirdar J, Thomas B, Ioachim HL, Litzky LA, Brambilla EM, Minna JD, Gazdar AF (1998) Comparison of molecular changes in lung cancers in HIV-positive and HIV- indeterminate subjects. JAMA 279: $1554-1559$

Wistuba II, Behrens C, Virmani AK, Mele G, Milchgrub S, Girard L, Fondon JW, Garner HR, McKay B, Latif F, Lerman MI, Lam S, Gazdar AF, Minna JD (2000) High resolution chromosome $3 p$ allelotyping of human lung cancer and preneoplastic/preinvasive bronchial epithelium reveals multiple, discontinuous sites of $3 p$ allele loss and three regions of frequent breakpoints. Cancer Res 60: 1949-1960

Wistuba II, Behrens C, Virmani AK, Milchgrub S, Syed S, Lam S, Mackay B, Minna JD, Gazdar AF (1999a) Allelic losses at chromosome 8p21-23 are early and frequent events in the pathogenesis of lung cancer. Cancer Res 59: $1973-1979$

Wistuba II, Tang M, Maitra A, Alvarez H, Troncoso P, Pimentel F, Gazdar AF (2001) Genome-wide allelotyping analysis reveals multiple sites of allelic loss in gallbladder carcinoma. Cancer Res 61: 3795-800

Wistuba II, Behrens C, Milchgrub S, Bryant D, Hung J, Minna JD, Gazdar AF (1999b) Sequential molecular abnormalities are involved in the multistage development of squamous cell lung carcinoma. Oncogene 18: 643-650

Wistuba II, Sugio K, Hung J, Kishimoto Y, Virmani, AK, Roa I, AlboresSaavedra J, Gazdar AF (1995) Allele specific mutations involved in the pathogenesis of endemic gallbladder carcinoma in Chile. Cancer Res 55: $2511-2515$ 